\title{
Aplicação da ultrassonografia colorida doppler em programas de transferência de embriões equinos
}

\author{
Potential use of doppler ultrasound in equine embryo transfer programs
}

\author{
Jair Camargo Ferreira ${ }^{\mathrm{I}}$ Cezinande de Meira'
}

\section{- REVISÃO BIBLIOGRÁFICA -}

\section{RESUMO}

Os programas comerciais de transferência de embriões em éguas existem por mais de três décadas e são hoje uma das biotécnias mais utilizadas na reprodução assistida de equinos. $O$ exame ultrassonográfico nos períodos pré e póscobertura de doadoras de embriões, assim como a avaliação de receptoras no momento da inovulação é de vital importância para o êxito de um programa de transferência de embriões. A ultrassonografia Doppler é uma técnica não-invasiva que permite a avaliação em tempo real da hemodinâmica do trato reprodutivo de animais de grande porte. Por fornecer detalhes anatômicos e informações imediatas sobre a fisiologia do fluxo sanguíneo de tecidos e órgãos, o exame doppler permite a avaliação do potencial ovulatório de folículos e do status funcional de corpo lúteo e útero, além de ser uma técnica auxiliar para o diagnóstico de distúrbios na hemodinâmica do sistema reprodutivo. Alterações na perfusão sanguínea dos futuros folículos dominantes são detectáveis em diferentes fases de seu desenvolvimento, como na divergência folicular e no período pré-ovulatório. A partir dessas informações, é possível determinar o princípio da atividade sexual e o momento ideal para o início de tratamentos superovulatórios e indutores de ovulação, assim como o momento mais apropriado para a realização de coberturas de éguas doadoras de embriões. A avaliação através do modo-Doppler do corpo lúteo e útero de éguas receptoras é também um instrumento auxiliar para a seleção de animais com perfil sérico de progesterona e ambiente uterino adequados para a sobrevivência do embrião e manutenção da gestação. Essa técnica pode ser útil ainda ao se avaliar a interação concepto-maternal. Apesar da aplicabilidade da tecnologia doppler dentro de programas de transferência de embriões, novos estudos visando a determinar padrões de normalidade $e$ posterior caracterização de distúrbios de fluxo sanguíneo de trato reprodutivo ainda se fazem necessários.
Palavras-chave: égua, ultrassonografia doppler, doadora, receptora, gestação.

\section{ABSTRACT}

Embryo transfer in mares has been commercially used for more than three decades and it is one of most frequently applied biotechnologies on equine reproduction. Ultrasonic doppler exam of donors during the pre and post-breeding and recipients evaluation at the embryo transfer moment is essential for embryo transfer program success. Doppler ultrasonography is a non-invasive technic that allows real-time evaluation of the reproductive system hemodynamics in large animal. For providing anatomical details and blood-flow physiologic information of vessels and tissues, doppler exam can be used for ovulation prognostication, and to evaluate uterine and luteal functional status. Additionally, it can serve as a diagnostic aid of hemodynamic disturbance in reproductive system. Vascularity changes of future ovulatory follicles precede the diameter deviation. Based on this, it is possible to estimate the beginning of the breeding season and the best moment to initiate superovulatory treatments. Moreover, follicular blood flow can be used to decide the most appropriated moment for ovulation induction and breeding of donors mares. Uterine and luteal evaluation using Doppler-mode in mares can be useful to select embryo recipients with satisfactory progesterone production and adequate uterine vascularity for embryo development and maintenance of pregnancy. The maternal recognition of pregnancy also can be evaluated. Although the applicability of the Doppler technology in embryo transfer programs, additional studies are necessary to determine the standards of normality and to characterize different bloodflow disturbances of the reproductive system.

Key words: mare, doppler ultrasonography, donor, recipient, gestation.

IPrograma de Pós-graduação em Medicina Veterinária, Departamento de Reprodução Animal e Radiologia Veterinária, Faculdade de Medicina Veterinária e Zootecnia (FMVZ), Universidade Estadual Paulista Júlio de Mesquita Filho (Unesp),18608-210, Botucatu, SP, Brasil. Email: jaircfvet@yahoo.com.br. Autor para correspondência.

IIFMVZ, UNESP, Botucatu, SP, Brasil. 


\section{INTRODUÇÃO}

A técnica de transferência de embriões (TE) não-cirúrgica em éguas foi descrita pela primeira vez por OGURI \& TSUTSUMI (1972). Desde então, ela é utilizada comercialmente em diversos países, sendo uma das principais biotécnicas da reprodução assistida de equinos. Dentre as diversas vantagens de seu emprego, pode-se citar a utilização de doadoras jovens, o aumento da produtividade égua/ano, a obtenção de produtos de éguas incapazes de conduzir a gestação a termo por problemas adquiridos e a possibilidade de manter éguas de performance em atividade ao longo do ano (RIERA, 2009). Com o intuito de aproveitar ao máximo o potencial de animais de alto valor genético, a TE vem sendo associada a outras técnicas de reprodução assistida como a criopreservação de embriões, superovulação (SOV), injeção intracitoplasmática de espermatozóides (ICSI) e até clonagem(MCKINNON \& SQUIRES, 2007).

Com o advento da ultrassonografia (US) colorida Doppler na reprodução equina, foi possível reavaliar conceitos antes considerados definitivos quanto à fisiologia da reprodução. Essa técnica demonstrou ser efetiva e prática para a avaliação não invasiva e em tempo real da perfusão vascular do trato reprodutivo de equinos (GINTHER, 2007). A presente revisão tem como objetivos: 1 ) descrever as principais alterações na fisiologia do fluxo sanguíneo do trato reprodutivo de éguas gestantes e não-gestantes; 2) discorrer sobre a possível aplicação da Dopplervelocimetria como técnica auxiliar na avaliação de éguas doadoras e receptoras de embriões, assim como na viabilidade do concepto.

\section{Princípios da ultrassonografia doppler}

Em 1842, o pesquisador austríaco Johann Christian Doppler descreveu o efeito Doppler como a alteração na frequência de uma onda emitida ou refletida por um objeto que se encontra em movimento em relação ao observador (CERRI et al., 1998a). Seguindo este princípio, a US Doppler baseia-se na movimentação das hemácias em relação ao transdutor, de forma que alterações de velocidade e sentido do fluxo sanguíneo são representados por imagens com específicas cores e tonalidades (GINTHER, 2007).

A técnica de ultrassonografia Doppler apresenta três modos de ação distintos: modo-B, modoDoppler e modo-Espectral. O modo-B utiliza escalas de cinza e é primordialmente empregado para a identificação anatômica de estruturas a serem avaliadas posteriormente pelos modos coloridos. Através do modo-Doppler, subdividido em funções color e power- flow, é possível estimar a perfusão sanguínea tecidual, levando-se em consideração a percentagem de tecido com pixels coloridos durante o exame. Já o modo-espectral fornece valores exatos de velocidades de fluxo sanguíneo e índices Doppler vasculares e teciduais (GINTHER \& MATTHEW, 2004). A adequada mensuração das velocidades de fluxo sanguíneo durante o exame espectral é dependente da correta angulação (ângulo Doppler) entre o transdutor e o vaso sanguíneo avaliado (GINTHER, 2007). Devido a grande tortuosidade das artérias presentes no mesométrio, ovários e útero, não é possível determinar os seus respectivos ângulos Doppler. Entretanto, como os índices de resistência (RI) e pulsatilidade (PI) não sofrem influência dessa angulação, eles são indicados para a avaliação espectral do trato reprodutivo de éguas (SILVA et al., 2005; FERREIRA et al., 2010). RI e PI apresentam correlação negativa com a perfusão vascular do tecido irrigado pela artéria em questão, ou seja, quanto menores RI e PI maior será a perfusão vascular no tecido suprido por aquele vaso(GINTHER, 2007).

Os primeiros estudos com US Doppler baseavam-se em informações obtidas nas grandes artérias (artérias ovariana, uterina e vaginal) responsáveis pela suplementação sanguínea do trato reprodutivo (BOLLWEIN et al., 1998). Contudo, os estudos recentes tendem a realizar exames diretamente no tecido alvo, como, por exemplo, endométrio, parede folicular e corpo lúteo (CL), ou em vasos próximos (SILVA et al., 2005; SILVA \& GINTHER, 2006; GINTHER et al., 2008; FERREIRA et al., 2008; FERREIRA et al., 2010).

Detecção do início da atividade reprodutiva

A detecção precoce do início da atividade cíclica é uma ferramenta valiosa no manejo reprodutivo de equinos, pois possibilita uma maior produção de embriões ao longo da estação de monta e evita a realização de coberturas e tratamentos indutores de ovulação desnecessários. Através da US Doppler, é possível determinar o potencial ovulatório de folículos dominantes durante o período de transição de primavera (ACOSTA et al., 2004a).

A transição de primavera é caracterizada pela presença de ondas foliculares menores e/ou ondas maiores anovulatórias. Acosta et al. (2004a) demonstraram que o diâmetro máximo e a taxa de crescimento folicular não são bons indicadores do status ovulatório de folículos durante o período de transição, ao contrário da vascularidade da parede folicular. Folículos dominantes ovulatórios apresentam um aumento progressivo da vascularidade a partir da divergência folicular, enquanto que folículos anovulatórios apresentam baixa vascularidade 
independente de seu diâmetro. Acredita-se que esta vascularidade diminuída de folículos maiores anovulatórios esteja relacionada com a baixa concentração sérica de LH durante o período de transição. Esta deficiência de LH resultaria na baixa concentração de fatores intra-foliculares, como estradiol, fator de crescimento endotelial vascular (VEGF) e inibina (GINTHER et al., 2003). As baixas concentrações de estradiol e inibina resultam no aumento na concentração sérica de FSH e contínuo crescimento folicular, enquanto que a baixa concentração de VEGF ocasiona distúrbios na angiogênese das células da parede folicular (GINTHER, 2007).

\section{Tratamento superovulatório}

Estudo realizado em éguas cíclicas, ou seja, que apresentam atividade reprodutiva que culminem na ovulação, descreve que o futuro folículo ovulatório apresenta um aumento progressivo de vascularidade um dia antes da divergência até o dia da ovulação (OV). Já folículos subordinados apresentam baixo fluxo sanguíneo ao longo de todo o ciclo (ACOSTA et al., 2004b).

Dentre as técnicas alternativas utilizadas em programas de TE em equinos, o uso de tratamentos superovulatórios possibilita o aumento no número de embriões viáveis/ciclo estral destinados para transferência a fresco e/ou criopreservação (MCKINNON \& SQUIRES, 2007). Orlandi (2008) demonstrou que ao iniciar a SOV próximo ao momento da divergência folicular (20-23mm) é possível reduzir o tempo de tratamento mantendo a taxa de OV/égua. Como a divergência é precedida por alterações na vascularidade folicular, é provável que a US Doppler possa ser uma ferramenta útil para determinar o momento ideal para o início de tratamentos superovulatórios. Entretanto, estudos a esse respeito ainda se fazem necessários.

Predição de ovulação e eficácia de tratamentos indutores

Ao inseminar éguas com sêmen congelado é imprescindível determinar a iminência da OV para que o depósito do sêmen seja realizado o mais próximo possível do momento da liberação do oócito (SAMPER et al., 2007). A US Doppler acrescenta novas informações quanto à maturação folicular final e a proximidade da $\mathrm{OV}$, podendo ser utilizada para o acompanhamento do desenvolvimento folicular de éguas doadoras de embriões, principalmente quando se utiliza sêmen congelado.

De acordo com PIERSON \& GINTHER(1985) e GASTAL et al. (1997, 1998, 2006a), é possível predizer o momento da ovulação através da US modo-B, levando- se em consideração alterações morfoecogênicas do folículo dominante. Adicionalmente, a US doppler surge com um método complementar para predizer com maior precisão o momento da ovulação em animais de grande porte.

Como já citado anteriormente, o futuro folículo ovulatório apresenta um aumento progressivo na vascularidade até o dia da OV (ACOSTA et al., 2004b). Estudo recente realizado por GASTAL et al. (2006b) avaliou as variações de fluxo sanguíneo folicular durante as 36 horas que antecedem a OV espontânea ou induzida com hCG. Foi demonstrado que, em casos de OV espontânea, o fluxo sanguíneo folicular é máximo (80 a 90\% da parede folicular apresenta pontos coloridos durante o exame ultrassonográfico) entre as horas 36 e 12 que antecedem a OV, e que a quantidade de pixels coloridos Doppler diminui abruptamente durante as últimas quatro horas pré-OV. Já a vascularidade do folículo dominante de éguas tratadas com hCG aumenta progressivamente durante as primeiras 24 horas pós-tratamento e diminui nas quatro últimas horas pré-OV, de forma semelhante ao observado em casos de OV espontânea.

Considerando que o folículo de éguas induzidas com hCG apresentam um aumento na perfusão sanguínea nas primeiras 24 horas e ovulam em média entre 36 e 42 horas pós-tratamento (GASTAL et al., 2006b), a US Doppler permite a avaliação precoce da eficácia de tratamentos indutores de ovulação.

Diagnóstico auxiliar de distúrbios na ovulação

Evacuação é o fenômeno observado durante a ovulação consequente à ruptura da parede folicular, permitindo o extravasamento do fluído folicular e liberação do oócito (GINTHER, 1995). As alterações hemodinâmicas observadas durante esse fenômeno já foram caracterizadas anteriormente.

Evacuação septada é um fenômeno atípico observado em éguas. Com duração superior a três horas (GINTHER et al., 2006a), sua incidência ao longo da estação de monta é de aproximadamente 19\% (CARNEVALE et al., 1988). A etiologia dessa anomalia ainda é desconhecida, entretanto, sabe-se que distúrbios na hemodinâmica folicular no período préovulatório estão envolvidos (GINTHER, 2007). GINTHER et al. (2006a) descreveram que folículos que evacuam lentamente, com presença de septos e compartimentos, apresentam proeminente vascularização ao longo de toda sua circunferência, inclusive na região do ápice folicular. Já durante uma evacuação normal, a perfusão sanguínea diminui poucas horas antes da OV e concentra-se na base do folículo (GASTAL et al., 2006b) 
Folículo hemorrágico anovulatório (FHA) é a estrutura decorrente da falha na ovulação, seguida pela formação de um hematoma no antro folicular de éguas (GINTHER \& PIERSON, 1984). De forma semelhante ao observado em casos de ovulação septada, o futuro FHA apresenta intensa vascularização ao longo de sua circunferência (GINTHER et al., 2006b). Por resultar em ciclos inférteis e apresentar elevada incidência de repetibilidade (GINTHER, 2007), o diagnóstico precoce e prévio à cobertura de FHAs pode ser benéfico em um programa de TE, já que, dessa maneira, evitam-se gastos desnecessários com tratamentos indutores de OV e transporte de sêmen ao longo da estação de monta.

\section{Avaliação do status funcional do CL}

THOWNSON et al. (1989) descreveram a ocorrência frequente de dois tipos de CL em éguas: cavitários e não-cavitários. O mesmo estudo demonstrou não existir relação entre a funcionalidade luteal e a presença ou ausência da cavidade. De acordo com GINTHER (1995), estudos variados utilizando a US modo-B demonstraram resultados divergentes quanto à relação existente entre a morfoecogenicidade luteal e a concentração sistêmica de progesterona em éguas.

Levando-se em consideração que o desenvolvimento, regressão e funcionalidade luteal estão estritamente relacionados com o rápido desenvolvimento e regressão de um extenso sistema vascular local, era de se esperar que a US Doppler fosse de grande valia para a avaliação do status funcional do CL. Estudo recente caracterizou a relação existente entre a perfusão sanguínea com o desenvolvimento estrutural e funcional do CL (GINTHER et al., 2007). Após a evacuação folicular, a perfusão sanguínea do CL aumenta progressivamente até envolver praticamente toda sua circunferência no sexto dia pós-OV. Durante este período, a progesterona (P4) também aumenta progressivamente. Entre D8 e D14, o perfil sérico de P4 e perfusão vascular luteal apresentam valores máximos. Já durante a luteólise, a vascularidade do CL diminui, fenômeno acompanhado pela diminuição na concentração de P4. Em bovinos, a luteólise é antecedida por um aumento agudo na perfusão sanguínea luteal (ACOSTA et al., 2002; MIYAMOTO et al., 2005). Em éguas, esse fenômeno não é observado (GINTHER et al., 2008).

A manutenção da gestação em éguas, assim como nas demais espécies domésticas, requer constante síntese de progesterona (ALLEN \& STEWART, 2001). A administração de P4 é frequentemente empregada em programas de reprodução equina com o intuito de prevenir a morte embrionária/fetal precoce devido à insuficiência luteal (ALLEN, 2001). Considerando a íntima relação entre a perfusão vascular do CL e a concentração sérica de P4, a US Doppler pode ser empregada no momento da inovulação do embrião para avaliar a funcionalidade do CL e, dessa forma, selecionar a receptora com perfil progesterônico mais adequado e/ou para determinar se a suplementação com P4 exógena se faz necessária.

Éguas receptoras acíclicas, ou seja, que não apresentam atividade sexual, são normalmente suplementadas com P4 exógena desde o momento da inovulação do embrião até aproximadamente 120 dias de gestação, quando a gestação é completamente placentária-dependente (ALLEN, 2001). Segundo GRECO et al. (2008), tais receptoras desenvolvem CL suplementares. Entretanto, ainda é incerto se os CLs suplementares são capazes de manter a gestação na ausência de um CL primário. As características de perfusão vascular dos CLs suplementares e sua relação com o perfil sérico de P4 ainda são desconhecidos. Uma vez determinada tal relação, a ultrassonografia Doppler poderá ser utilizada visando interromper o tratamento suplementar com $\mathrm{P} 4$, tendo como base a vascularidade dos CLs.

\section{Avaliação do status funcional uterino}

A seleção de receptoras é tida como um dos fatores mais importantes para o sucesso de um programa de TE. Dentre os diversos elementos a serem considerados, destaca-se a capacidade do útero em conduzir a gestação a termo (MCKINNON \& SQUIRES, 2007). A avaliação do útero de éguas doadoras nos períodos pré e pós-cobertura também é importante, no intuito de não comprometer a viabilidade dos espermatozóides, evitar a luteólise precoce e a formação de um ambiente inóspito para o embrião até o momento de sua transferência para a égua receptora.

Considerando distúrbios vasculares como potenciais causadores de alterações degenerativas do endométrio (FERREIRA et al., 2008), e que essas alterações, por sua vez, estão intimamente relacionadas com casos de subfertilidade em éguas (DOIG et al. 1981; TANNUS \& THUN,1995; LEIDL et al., 1987), a US Doppler é uma importante ferramenta para o estudo da viabilidade endometrial, assim como para a interação concepto-maternal. Ferreira et al. (2008) demonstraram que a US Doppler é efetiva na avaliação da perfusão sanguínea do útero de éguas que apresentam ou não cistos uterinos. Neste estudo, foi observado que regiões císticas apresentam menor perfusão sanguínea do que regiões adjacentes não-císticas, sugerindo que distúrbios vasculares estão relacionados com a formação de cistos uterinos. Com etiopatogenia incerta 
até o momento, é provável que o fluxo sanguíneo anormal do útero contribua para a formação de cistos, devido ao pobre retorno venoso e degeneração arterial presentes naquele segmento uterino (SCHOON et al., 1999).

De acordo com SILVA et al. (2005), éguas não-gestantes apresentam vascularidade endometrial baixa e constante durante os 15 primeiros dias pós-OV. Aparentemente, éguas que acumulam fluído intrauterino nos quatro dias subsequentes à cobertura apresentam maior perfusão sanguínea uterina e menores valores de RI e PI mesometrial (FERREIRA et al., dados não-publicados). Dessa forma, é provável que, em futuro próximo, a US Doppler possa ser empregada no diagnóstico auxiliar de endometrites pré e pós-cobertura.

Diagnóstico de gestação e viabilidade embrionária Mudanças na hemodinâmica do sistema reprodutivo durante a gestação inicial já foram descritas tanto em seres humanos como em equinos. Em humanos, a receptividade do endométrio, a implantação e o desenvolvimento do embrião são dependentes da adequada irrigação uterina (STERZIK et al., 1989; CHIEN et al., 2004). Durante a fase de mobilidade embrionária em éguas, alterações na perfusão vascular endometrial ocorrem de forma localizada em associação com mudanças da posição do concepto (SILVA et al., 2005). De acordo com o mesmo estudo, éguas gestantes e não-gestantes apresentam similar e baixa vascularidade endometrial nos oito primeiros dias pósovulação. Entretanto, um aumento da perfusão vascular é notado a partir do dia 11 no endométrio de ambos os cornos uterinos de éguas gestantes. Um pronunciado aumento no corno que contém a vesícula, quando comparado com o corno contra-lateral, também é observado. Este aumento é ainda mais acentuado no tecido endometrial que está em contato direto com a vesícula embrionária. Estudo recente quantificou os índices doppler (RI e PI) uterinos de éguas durante os primeiros 20 dias da gestação. Ambos os índices diminuíram progressivamente com o avançar da gestação (FERREIRA et al., 2010).

Ao contrário do observado por SILVA et al. (2005), ao avaliar o útero de éguas gestantes, FERREIRA et al. (2010) observaram um aumento da perfusão vascular entre D3 e D6 pós-OV. O aumento na perfusão vascular uterina entre D3 e D6 pós-OV foi acompanhado pela diminuição nos valores de RI e PI mesometriais. Perfusão vascular uterina e índices doppler mesometriais aumentaram e diminuíram, respectivamente, a partir de D12. A visualização desta alteração na hemodinâmica uterina pode ser útil para o diagnóstico precoce da gestação, assim como para determinar o momento ideal para a coleta de embriões visando à criopreservação.

Utilizando a US Doppler modo-espectral, CHEN \& STOLLA (2006) desenvolveram o índice uterino (IU) com o intuito de prever a morte embrionária em éguas. De acordo com os autores, IU menor que cinco é um indicativo de morte embrionária evidente nas próximas 24 horas, enquanto que éguas com IU maior que 10 não apresentaram nenhum distúrbio hemodinâmico aparente.

Fase intermediária e final da gestação Informações sobre a aplicação da US Doppler nas fases intermediária e final da gestação em éguas ainda são escassas na literatura. Os estudos realizados até o momento visaram descrever anatomicamente o sistema vascular de feto e útero gestante (GINTHER, 2007). Em humanos, o exame doppler apresenta uma vasta aplicação na obstetrícia. Dentre as indicações da Dopplervelocimetria, temos o diagnóstico de diversas patologias e distúrbios da gestação como, por exemplo, doença hipertensiva específica da gestação, neoplasia trofoblástica gestacional, tumores uterinos, accretismo placentário, ameaça de abortamento, cistos ovarianos, insuficiência lútea, alterações da vitalidade, malformação fetal, entre outros (CERRI et al., 1998b).

\section{Considerações finais}

A espécie equina foi uma das mais utilizadas pelos pesquisadores pioneiros no desenvolvimento da ultrassonografia transretal para o exame in vivo do trato reprodutivo. No início dos anos 80, essa técnica revolucionou o diagnóstico e o monitoramento de eventos reprodutivos biológicos e patológicos em programas de reprodução assistida. Hoje, informações imediatas sobre a fisiologia do fluxo sanguíneo de vasos, tecidos e órgãos do sistema reprodutivo podem ser obtidas através da ultrassonografia Doppler. Por meio desse novo conhecimento, conceitos antes considerados definitivos quanto à fisiologia da reprodução equina estão sendo reavaliados.

Em programas de transferência de embriões, a ultrassonografia Doppler apresenta um amplo potencial de uso no intuito de otimizar o emprego tanto de éguas doadoras como receptoras. Atualmente, dentre suas diversas aplicações, destacam-se a possibilidade de predizer o início da ciclicidade e determinar o momento ideal para a cobertura de doadoras de embriões, além de ser útil na avaliação do potencial em manter a gestação de receptoras. Contudo, muitos estudos ainda são necessários para determinar 
parâmetros de normalidade, assim como para descrever patologias reprodutivas.

\section{REFERÊNCIA}

ACOSTA, T.J. et al. Local changes in blood flow within the early and midcycle corpus luteum after prostaglandin $\mathrm{F}$ (2 alpha) injection in the cow. Biology of Reproduction, v.66, p.651658, 2002. Disponível em: <http://www.biolreprod.org/content/ 66/3/651.long>. Acesso em: 28 mar. 2011. doi: 10.1095/ biolreprod66.3.651.

ACOSTA, T.J. et al. Aberrant blood flow area and plasma gonadotropin concentrations during the development of dominant-sized transitional anovulatory follicles in mares. Biology of Reproduction, v.71, p.637-642, $2004 \mathrm{a}$. Disponível em: <http://www.biolreprod.org/content/71/2/ 637.long>. Acesso em: 28 mar. 2011. doi: 10.1095/ biolreprod.104.028498.

ACOSTA, T.J. et al. Differential blood flow changes between the future dominant and subordinate follicles precede diameter changes during follicle selection in mares. Biology of Reproduction, v.71, p.502-507, 2004b. Disponível em: <http:/ /www.biolreprod.org/content/71/2/502.long>. Acesso em: 28 mar. 2011. doi: 10.1095/biolreprod.104.027896.

ALLEN, W.R.; STEWART, F. Equine placentation. Reproduction, Fertility and Development, v.13, p.623634, 2001. Disponível em: <http://www.publish.csiro.au/ index.cfm?paper=RD01063>. Acesso em: 28 mar. 2011. doi: 10.1071/RD01063.

ALLEN, W.R. Luteal deficiency and embryo mortality in the mare. Reproduction in Domestic Animals, v.36, p.121131, 2001. Disponível em: <http://onlinelibrary.wiley.com/ doi/10.1046/j.1439-0531.2001.00312.x/pdf>. Acesso em: 28 mar. 2011. doi: 10.1046/j.1439-0531.2001.00312.x.

BOLLWEIN, H. et al. Transrectal color Doppler sonography of the a. uterine. Theriogenology, v.49, p.1483-1488, 1998.

CERRI, G.G. et al. Princípios básicos e instrumentação. In: Cerri, G.G. (Ed) Doppler. São Paulo: Sarvier, 1998a. Cap.1, p.1-14.

CERRI, G.G. et al. Aplicação do Doppler em obstetrícia. In: Cerri, G.G. (Ed) Doppler. São Paulo: Sarvier, 1998b. Cap.9,p.183-219.

CARNEVALE, E.M. et al. Ultrasonographic characteristics of the preovulatory follicle preceding and during ovulation in mares. Journal of Equine Veterinary Science, v.8, p.428431, 1988.

CHEN, Y.H.; STOLLA, R. Using uterine index to diagnose embryonic death in mares. Journal of Equine Veterinary Science, v.26, p.219-224, 2006. Disponível em: <http:// www.sciencedirect.com/science?>. Acesso em: 28 mar. 2011. doi: $10.1016 /$ j.jevs.2006.03.004.

CHIEN, L.W. et al. Assessment of changes in utero-ovarian arterial impedance during the peri-implantation period by Doppler sonography in women undergoing assisted reproduction. Ultrasound in Obstetrics and Gynecology, v.23, p.496500, 2004. Disponível em: <http://onlinelibrary.wiley.com/ doi/10.1002/uog.975/pdf>. Acesso em: 28 mar. 2011. doi: 10.1002/uog.975

DOIG, P.A. et al. The use of endometrial biopsy in the infertile mare. Canadian Veterinary Journal, v.22, p.72-76, 1981.

FERREIRA J.C. et al. Uterine blood flow and perfusion in mares with uterine cysts: effect of the size of the cystic area and age. Reproduction, v.135, p.541-550, 2008. Disponível em: <http://www.reproduction-online.org/cgi/reprint/135/4/ 541>. Acesso em: 28 mar. 2011. doi: 10.1530/REP-07-0447.

FERREIRA J.C. et al. Uterine vascular perfusion and spectralDoppler measurements during the early gestation in mares: news concepts of evaluation. In: INTERNATIONAL SYMPOSIUM ON EQUINE REPRODUCTION, 10., 2010, Lexington, KY, USA. Proceedings... Lexington:Elsevier, 2010. V.1. p.281-283.

GASTAL, E.L. et al. Serrated granulosa and other discrete ultasound indicators of impending ovulation in mares. Journal Equine Veterinary Science, v.26, p.67-73, $2006 \mathrm{a}$. Disponível em: <http://www.sciencedirect.com/science>. Acesso em: 28 mar. 2011. doi:10.1016/j.jevs.2005.12.005.

GASTAL, E.L. et al. Relationships of changes in B-mode echotexture and colour-Doppler signals in the wall of the preovulatory follicle to changes in systemic oestradiol concentrations and the effects of human chorionic gonadotrophin in mares. Reproduction, v.131, p.699-709, 2006b. Disponível em: <http://www.reproduction-online.org/ cgi/reprint/131/4/699>. Acesso em: 28 mar. 2011. doi: 10.1530/ rep.1.01011.

GASTAL, E.L. et al. The suitability of echotexture characteristics of the follicular wall for identifying the optimal breeding day in mares. Theriogenology, v50 p.1025-1038, 1998.

GASTAL, E.L. et al. Role of diameter differences among follicles in selection of a future dominant follicle in mares. Biology of Reproduction, v.57, p.1320-1327, 1997. Disponível em: <http://www.biolreprod.org/content/57/6/1320.long>. Acesso em: 28 mar. 2011. doi: 10.1095/?biolreprod57.6.1320.

GINTHER, O.J.; PIERSON, R.A. Ultrasonic anatomy of the equine ovaries. Theriogenology. v.21, p.471-483, 1984.

GINTHER, O.J.; MATTHEW, D.U. Doppler ultrasound in equine reproduction: principles, techniques, and potential. Journal of Equine Veterinary Science, v.24 p.516-526, 2004. Disponível em: <http://www.sciencedirect.com/science?>. Acesso em: 28 mar. 2011. doi: 10.1016/j.jevs.2004.11.005.

GINTHER, O.J. et al. Mechanism of follicle deviation in monovular farm species. Animal Reproduction Science, v.78, p.239-257, 2003. Disponível em: <http:// www.sciencedirect.com/science?>. Acesso em: 28 mar. 2011. doi: 10.1016/S0378-4320(03)00093-9.

GINTHER, O.J. et al. Luteal blood flow and progesterone production in mares. Animal Reproduction Science, v.99, p.213-220, 2007. Disponível em: http://www.sciencedirect.com/ science?>. Acesso em: 28 mar. 2011. doi:10.1016/ j.anireprosci.2006.05.018. 
GINTHER, O.J. et al. Characterisation of pulses of 13,14dihydro-15-keto-PGF2alpha (PGFM) and relationships between PGFM pulses and luteal blood flow before, during, and after luteolysis in mares. Reproduction, Fertilility and Development, v.20, p.684-693, 2008. Disponível em: <http:/ /www.publish.csiro.au/?act=view_file\&file_id=RD08077.pdf $>$. Acesso em: 28 mar. 2011. doi: 10.1071/RD08077.

GINTHER, O.J. Follicles. In: (Ed). Ultrasonic imaging and animal reproduction: horses. Cross Plains: Equiservices, 1995. p.43-66.

GINTHER, O.J. Ultrasonic imaging and animal reproduction: Color-Doppler ultrasonography. Cross Plains: Equiservices, 2007. 258p.

GINTHER, O.J. et al. Spatial relationships between serrated granulosa and vascularity of the preovulatory follicle and developing corpus luteum. Journal of Equine Veterinary Science, v.27, p.20-27, 2006a. Disponível em: <http:// www.sciencedirect.com/science?>. Acesso em: 28 mar. 2011. doi: 0.1016/j.jevs.2006.11.012.

GINTHER, O.J. et al. Conversion of a viable preovulatory follicle into a hemorrhagic anovulatory follicle in mares. Animal Reproduction, v.3, p.29-40, 2006b Disponível em: <http://www.cbra.org.br/pages/publicacoes/animalreproduction/ issues/download/v3n1/AR123\%20Ginther\%20pag29-40.pdf> Acesso em: 28 mar. 2011.

GRECO, G.M. et al. Efeito da interrupção do tratamento com progesterona em éguas receptoras acíclicas apresentando a formação de corpos lúteos acessórios. Acta Scientiae Veterinariae, v.36, p.590, 2008.

LEIDL, W. et al. Endometrial cysts in the mare. 2. Clinical studies: occurrence and significance. Tierärztliche Praxis , v.15, p.281-289, 1987.

McKINNON A.O.; SQUIRES E.L. Embryo transfer and related technologies. In: RUDOLPH, P.; GOWER, J. (Ed.). Current therapy in equine reproduction. Missouri: Saundersd Elseviers, 2007. p.319-334.

MIYAMOTO, A. et al. Blood flow: a key regulatory component of corpus luteum function in the cow. Domest Animal Endocrinology., v.29, p.329-339, 2005. Disponível em: <http://www.sciencedirect.com/science?>. Acesso em: $28 \mathrm{mar}$. 2011. doi: 10.1016/j.domaniend.2005.03.011.

OGURI, N.; TSUTSUMI, Y. Non-surgical recovery of equine eggs, and an attempt at non-surgical egg transfer in horses.
Journal of Reproduction and Fertility, v.31, p.187-195, 1972.

ORLANDI, C.M.B. Resposta ovariana e concentrações plasmáticas de FSH em éguas submetidas à aspiração folicular e tratadas com extrato de pituitaria eqüina (EPE). 2008. 68f. Tese (Doutorado em Reprodução Animal) - Faculdade de Medicina Veterinária e Zootecnia, Campus de Botucatu, Universidade Estadual Paulista Júlio de Mesquita Filho, Botucatu, SP.

PIERSON, R.A.; GINTHER, O.J. Ultrasonic evaluation of the preovulatory follicle in the mare. Theriogenology, v.24, p.359-368, 1985.

RIERA, F.L. Equine embryo transfer. In: RUDOLPH, P.; GOWER, J. (Ed.). Equine breeding management and artificial insemination. Missouri: Saunders Elsevier 2009. p.185-199.

SAMPER, J.C. et al. Insemination with frozen semen. In: RUDOLPH, P.; GOWER, J. (Ed.). Current therapy in equine reproduction. Missouri: Saunders Elseviers, 2007. p.285288.

SILVA, L.A. et al. Changes in vascular perfusion of the endometrium in association with changes in location of the embryonic vesicle in mares. Biology of Reproduction, v.72, p.755-761, 2005. Disponivel em: <http://www.biolreprod.org/ content/72/3/755.full.pdf + html>. Acesso em: 28 mar. 2011. doi: 10.1095/biolreprod.104.036384.

SILVA L.A.; GINTHER O.J. An early endometrial vascular indicator of completed orientation of the embryo and the role of dorsal endometrial encroachment in mares. Biology of Reproduction, v.74, p. 337-343, 2006. Disponível em: <http:/ /www.biolreprod.org/content/74/2/337.full.pdf + html>. Acesso em: 28 mar. 2011. doi: 10.1095/biolreprod.105.047621.

SCHOON, D. et al. Angioses in the equine endometrium pathogenesis and clinical correlations. Pferdeheilkunde, v.15, p.541-546, 1999.

STERZIK, K. et al. Doppler sonographic findings and their correlation with implantation in an in vitro fertilization program. Fertility and Sterility, v.52, p.825-828, 1989.

TANNUS, R.J.; THUN, R. Influence of endometrial cysts on conception rate of mares. Journal of Veterinary Medicine Series A, v.42, p.275-283, 1995. 